\section{CELLS}

\section{Mauling the Membrane}

from our Molecular Biology Correspondent

Whereas many antibiotics attack the biochemical vitals of the bacterial cell, others are known to interfere in one way or another with its controlled communication with the outside world. One such, whose mechanism of action has been notably obscure, is bacitracin. This is a peptide of curious structure, synthesized by some strains of a Bacillus. It contains, among other unusual features, a tract of residues joined in the form of a thiazoline ring. Its effects on cells are various, and all fatal. It evidently acts at both cell walls and membranes, and in the latter it induces permeability to potassium ions. It requires divalent metals for activity, and these are directly complexed by it.

One of the modes of function of bacitracin is to block the synthesis of a vital cell wall component, peptidoglycan. Stone and Strominger (Proc. US Nat. Acad. Sci., 68, 3223; 1971) have now uncovered a highly specific interaction of bacitracin with a membrane component, which seems to lie at the root of this effect. They have shown that in the presence of a divalent cation the bacitracin binds strongly to the membrane constituent, $\mathrm{C}_{55}$-isoprenyl pyrophosphate, so preventing its cleavage by a pyrophosphatase, with release of the lipid carrier implicated in the cell-wall synthetic cycle. Complex formation was demonstrated by a column chromatographic method, and was found to be prevented by EDTA and other chelating agents. The authors have built models to show how the antibiotic and its target molecule might interact. The possibility exists, of course, that the bacitracin disturbs the proper functioning of membranes by direct physical distortion, as well as causing lipid depletion.

A quite different kind of molecule, which is death to membranes, though afier a quite different fashion, involving apparently a cataclysmic structural disturbance of the bilayer, is the polyenic visual pigment constituent, retinal. Red cells, exposed to very low concentrations of retinal, lose their ability to retain potassium ions, and at slightly higher concentrations-still, however, in the micromolar range-haemolysis ensues. Azuma and Yoshizawa (Biochim. Biophys. Acta, 249, 135; 1971) have now shown that the efficacy of the retinal in both these respects depends on its stereoisomeric configuration. Both potassium release and haemolysis occur at markedly lower concentrations of the all-trans isomer than the 11-cis. Now it is the bent 11 -cis species that forms the prosthetic group of the visual pig- ment, rhodopsin. It undergoes photoisomerization, followed by hydrolysis from the protein, on exposure to light, and this event is accompanied by a membrane process-reportedly the release of potassium ions from the rod outer segment-which triggers nervous excitation. Now because rhodopsin is the major protein component of the rod outer segment membranes, it is an attractive proposition that the liberated prosthetic group might itself be the agency that brings about depolarization. The greater proclivity of the isomeric form, in which the molecule normally exists after its release to penetrate, or at any rate modify, the membrane, provides new fuel for this hypothesis.

Other polyenic species are familiar as membrane-active antibiotics, and like a number of peptide antibiotics, of which the best known is perhaps gramicidin $A$, they function, so the speculation goes, by forming aggregates that make tunnels in the bilayer, through which potassium ions can travel. By this means the active transport system is bypassed, with dire results for the cell. This proposed mechanism stands in contrast to that of the macrocyclic antibiotics, such as nonactin and valinomycin. These have the property of sequestering sodium or potassium ions, and are thought to operate as vehicles for carrying such ions through the hydrophobic barrier of the membrane. These conjectures have been strikingly upheld by the outcome of a critical experiment by Krasne, Eisenman and Szabo (Science, 174, 412; 1971).

It is known that in both real mem- branes and artificial bilayers, a change of temperature can bring about an internal phase transition, in which the paraffin chains pass from a close-packed hexagonal lattice to an essentially disordered fluid state. Ion-carriers would then be expected to operate only in the fluid phase, whereas the transport of ions through tunnels should be substantially unaffected by the condition of the hydrocarbon milieu. These predictions are precisely borne out by the measurements of Krasne et al. Bilayers were prepared by surface spreading of mixtures of glycerol esters. Melting, which can be observed by simple physical criteria, occurs, within a range of about $2^{\circ}$, at about $40^{\circ} \mathrm{C}$. The transport of ions across the bilayer was measured by conductivity, and in the presence of the potassium and the macrocyclic carriers, nonactin or valinomycin, this parameter was found to undergo a violent change, encompassing from three to four orders of magnitude, at the melting temperature. The effect is reversible. In the melted state, the conductivity is directly proportional, as it should be, to the concentration of carriers.

The conductivity mediated by gramicidin A displays altogether different behaviour. There is no sharp discontinuity on melting, and the conductivity is large in both states. Only the temperature coefficient of conductivity changes with the phase transition. This must be seen as strong support in favour of the two fundamentally different mechanisms of action of the two classes of antibiotics.

\title{
More X-rays from NP 0532
}

FURTHER observations of the pulsed Xray emission originating in the $\mathrm{Crab}$ pulsar NP 0532 are reported in next Monday's Nature Physical Science (January 24) by the group at the Centre d'Etudes Nucléaires de Saclay in France, who have looked in particular at the possibility of short term variations in the characteristics of the pulses. Their data come from two balloon flights carried out from southern France, the first on September 15, 1970, and the second on September 22, 1970. On each occasion the Crab Nebula was observed for three hours by detectors sensitive to the energy range $15 \mathrm{keV}$ to $150 \mathrm{keV}$.

As others have done, the French group have noticed that in the X-ray region the pulse that dominates the optical observations of the Crab pulsar in fact carries less energy than an interpulse that to the optical observers appears less significant. In the optical region the energy in the interpulse is about a half that in the main pulse, but at $0.5-9 \mathrm{keV}$ the energies are about equal. In the $15-150 \mathrm{keV}$ region covered by the French group the energy in the interpulse is 1.38 times that in the main pulse. But when they divided their results into two ranges of energy$15-50 \mathrm{keV}$ and $50-150 \mathrm{keV}$--they find that their data are not sufficient to trace the development of the interpulse with confidence.

They also reported the absence of evidence for significant changes with energy of the proportion of the X-ray emission from the direction of the $\mathrm{Crab}$ that is pulsed. According to the sum of their data, $22.16 \pm 2.54$ per cent of the $\mathrm{X}$-rays received from the $\mathrm{Crab}$ by their detectors are pulsed.

It is fortunate that the two flights bracketed a sudden change in the velocity of the wisps of material in the part of the Crab Nebula adjacent to the pulsar (September 20, 1970), but no significant changes in behaviour were detected on the second flight of the balloon. 